\title{
La Interfaz Cultural
}

\section{Martin Nakata}

Universidad Tecnológica de Sidney

El Doctor Martin Nakata es Director de la Casa de Aprendizaje Indígena Jumbuna y Presidente de la cátedra de Educación Indígena Australiana de la Universidad Tecnológica de Sidney. Es también investigador honorario de la Biblioteca Mitchell de Sidney.

Este artículo fue publicado originalmente en el año 2007 en The Australian Journal of Indigenous Education, 36, Supplement, 7-14. La traducción del artículo al castellano fue realizada por Débora McLauchlan. 


\title{
La Interfaz Cultural
}

\begin{abstract}
Resumen
Desde hace un tiempo vengo investigando y escribiendo sobre temas de educación indígena. Como todos, he visto bastantes buenos trabajos y aprendido mucho sobre lo que está sucediendo en Australia y a nivel internacional para mejorar los resultados de los alumnos indígenas en procesos de educación formal. Este artículo reúne algunas propuestas teóricas del trabajo que he venido realizando para el sector de educación superior en Australia durante la última década y aborda las recientes tendencias para incluir el conocimiento indígena en el currículo. Concluye con algunos principios básicos para ayudar a establecer los inicios de los Estudios indígenas australianos como una disciplina en el sector de la educación superior.
\end{abstract}

Palabras clave: educación intercultural, tendencia educacional, elaboración del programa educativo

\section{The Cultural Interface}

\begin{abstract}
For a while now I have been researching and writing about Indigenous education issues. Like you all, we have seen much good work and learnt much from what is going on across Australia and internationally to improve outcomes for Indigenous learners in formal education processes. This paper draws together some early theoretical propositions from the work I have been progressing for the higher education sector in Australia over the past decade and addresses recent trends to include Indigenous Knowledge into the curriculum. It concludes with some foundational principles to help establish early beginnings with Australian Indigenous studies as a discipline in the higher education sector.
\end{abstract}

Keywords: intercultural education, educational trends, curriculum development 


\section{Conocimiento Indígena}

$E^{\prime}$

renovado interés en sistemas de conocimiento y prácticas indígenas es generalizado a nivel global (Nakata, 2002). Este interés ha surgido, como lo describe Hoppers, en tiempos de “...nuevas configuraciones en las relaciones globales... [donde] la centralidad del conocimiento...[es] la divisa emergente en esa relación" (Hoppers, 2000, p. 283).

Por consiguiente, el discurso global sobre conocimiento indígena atraviesa una serie de intereses como el desarrollo sostenible, la biodiversidad y la conservación, intereses comerciales y corporativos. Circula a niveles internacional, nacional, estatal, regional y local en sectores gubernamentales, no gubernamentales y de comunidades indígenas, así como entre una serie de agendas intelectuales, públicas, privadas e indígenas. Se encuentra disperso entre varias categorías de actividad intelectual de Occidente tales como la investigación científica, el manejo de documentación y conocimiento, la protección de la propiedad intelectual, educación y salud. Está implicado de manera política, económica y social en la vida de millones de personas alrededor del mundo. Una búsqueda en Google del término conocimiento indígena puede darnos una idea del alcance global que tiene este campo.

Gran parte de esto surge a partir de consideraciones prácticas en contextos de desarrollo y de una relación práctica con el conocimiento local sobre el terreno y con diversos propósitos. El enfoque ecológico y ambiental, de manejo de recursos y las prácticas agrícolas refleja esto, así como también la preocupación por la conservación del conocimiento, el manejo de éste en medios digitales y la protección legal.

A través de un conjunto de intersecciones aún más complejo, esta nueva tendencia se mezcla con, se basa en, responde a, cuestiona y a su vez da forma a un conjunto de discursos ya institucionalizados por mucho más tiempo, con sus propias prácticas socio-históricas y discursivas, que incluyen el conocimiento objetivado de las sociedades y culturas indígenas y otra producción de conocimiento que explica nuestras posiciones históricas y contemporáneas.

En este espacio discursivo se encuentra lo que algunos llaman "el archivo", pero que yo prefiero ampliar y llamar el corpus, esto es, ese cuerpo de conocimientos, tanto histórico como en curso, producido por otros "acerca de nosotros" a través de una serie de textos intelectuales, gubernamentales e históricos. En el mundo académico, este corpus fue antes principalmente el 
dominio del campo de la antropología, pero ahora se encuentra presente en una serie de campos donde las inquietudes de los propios indígenas o sobre los pueblos y temas indígenas se intersectan con las disciplinas establecidas.

Poco a poco, a medida que los pueblos indígenas aportan al corpus sus propias narrativas, críticas, investigación y producción de conocimiento, se va incorporando a este una "voz indígena" discernible.

El surgimiento de un "conocimiento indígena" revaluado y revisado para su inclusión a contenidos de cursos y programas entusiasma a quienes lo ven como una fuente de conocimiento "no mediado":

- conocimiento que es claramente reconocido y diferenciado como "indígena" y está fuera o apartado del corpus occidental;

- conocimiento que puede constituir la base de la revisión de conceptos sobre indígenas y que pueden utilizarse para informar sobre programas y servicios más relevantes y útiles para los pueblos indígenas; y

- conocimiento que puede constituir la base de la revisión de teorías sobre la posición indígena contemporánea en la investigación social.

Pero debemos ser muy cuidadosos en esto. No todo es blanco o negro, y las cosas no se arreglan con solo añadir a la mezcla el componente indígena. Este es un espacio muy complejo y disputado.

\section{Espacios de Conocimiento Disputados}

En sus divergencias, los sistemas de conocimiento indígena y los científicos occidentales son considerados tan dispares en términos cosmológicos, epistemológicos y ontológicos, que se ha dicho que son "inconmensurables" (Verran, 2005) o "irreconciliables" (Russell, 2005).

Si bien estos conceptos filosóficos no se desarrollan en este artículo, es crucial que quienes tengan interés en introducir el conocimiento indígena en áreas del currículo entiendan estos conceptos y tengan algún grado de compresión de cómo las diferencias en esos niveles enmarcan la posible comprensión o incomprensión de aspectos del conocimiento indígena en niveles más superficiales.

La literatura al respecto está creciendo a nivel internacional y es un área que merece análisis y evaluación. Está surgiendo de una serie de intereses, sectores y proyectos alrededor del mundo. Va desde la crítica, a la cautela, la promoción, la teoría, la innovación y los ejemplos en la práctica (ver Agrawal, 1995a, 1995b, 1996; Battiste \& Youngblood, 2000; Christie, 2005; Ellen \& Harris, 1996; Gegeo \& Watson-Gegeo, 2001; Langton \& Ma Rhea, 2005; Russell, 2005; 
L. T. Smith, 1999; Verran, 2005). Es importante leer esta literatura de manera suficientemente crítica para así poder situar los argumentos de las diversas posiciones que existen en la literatura.

Las diferencias en estos niveles significan que, en el medio académico, no es posible traer el conocimiento indígena y colocarlo en el currículo sin problema alguno, como si fuera otro conjunto de datos de conocimiento occidental a instruir y evaluar. Los sistemas de conocimiento indígena y los sistemas de conocimiento occidentales parten de diferentes teorías de conocimiento que enmarcan,

quién puede ser un conocedor, qué puede conocerse, qué constituye conocimiento, fuentes de evidencia para construir el conocimiento, qué constituye la verdad, cómo se debe verificar la verdad, cómo se convierte la evidencia en verdad, cómo se deben extraer conclusiones válidas, el papel de las creencias en la evidencia, y otros temas relacionados. (Gegeo \& Watson-Gegeo, 2001, p.57)

Un sistema de conocimiento no puede verificar de manera legítima las "afirmaciones de la verdad" del otro por medio de sus propios estándares y justificaciones (Verran, 2005).

Las maneras en que estos sistemas "hacen" conocimiento son tan inconmensurables, que aún si se comprenden las diferencias epistemológicas y ontológicas y las interminables descripciones de éstos en varios lugares de producción de conocimiento, no podemos simplemente "hacer" conocimiento indígena en el currículo. En las universidades, nos adherimos a los mecanismos y prácticas institucionales de las tradiciones del conocimiento científico y occidental. En tal contexto, el aprendizaje y el "hacer" conocimiento está mediado por la organización del conocimiento disciplinario y sus prácticas discursivas y textuales.

En este contexto, se tratan representaciones del conocimiento indígena ya circunscritas por el idioma inglés y el posicionamiento discursivo de varias prácticas disciplinarias, que incluyen paradigmas científicos, concepciones históricas, conjuntos particulares de intereses, diversas posiciones teóricas, tecnologías de producción de textos, etc. Estas representaciones pueden describir de manera útil y cuidadosa la aplicación de diferentes enfoques de cada sistema de conocimiento a un punto central común para generar la comprensión. Pero la manera en que nosotros llegamos a conocer y comprender, debatir, criticar y analizar dentro de programas universitarios no es la manera en que los pueblos indígenas llegan a hacerlo en contextos locales.

Sin embargo, en el medio académico y sobre el terreno, el hablar de sistemas de conocimiento indígena, en lugar de culturas, logra introducir el 
conocimiento indígena, sus sistemas, sus expresiones y prácticas tradicionales a una relación con la ciencia occidental diferente a la que era posible a través de la antropología. Inicialmente, los estudios antropológicos de las sociedades y culturas indígenas eran usados para proporcionar evidencia a las teorías disciplinarias de desarrollo y evolución humanos (Peterson, 1990). La producción de conocimiento en este campo sirvió para racionalizar una serie de prácticas y actividades del capitalismo liberal por todo el mundo. Pero, a pesar de un gran cambio en la base de investigación durante el último siglo, toda la producción del conocimiento sobre pueblos indígenas aún opera dentro de un conjunto de relaciones sociales más amplio que racionaliza, justifica y trabaja para operacionalizar un complejo aparato de acciones burocráticas, administrativas y disciplinarias que continúan confinando las vidas de los indígenas.

Podemos sostener que el interés en sistemas de conocimiento indígena comienza en otro lugar, pero tenemos que reconocer que el conocimiento indígena está posicionado dentro de los campos discursivos de manera similar que cualquier otra producción de conocimiento "acerca de nosotros". Sin embargo, esto no desvirtúa el argumento de que el actual interés en el conocimiento indígena surge en un momento histórico distinto, en que los pueblos indígenas están mucho mejor posicionados dentro del orden político-legal y donde los temas de derechos humanos, soberanía, autodeterminación y de reparación histórica, proveen una mejor base para la afirmación de los intereses indígenas.

Por tanto, aunque aún son parámetros predominantemente no indígenas u occidentales los que dan forma al discurso del conocimiento indígena, sí se plantean preguntas relacionadas a su utilidad y valor en diversos contextos, incluido el indígena. En algunos contextos (pero por supuesto no en todos) esto focaliza la atención en prácticas sobre el terreno más colaborativas y generadas localmente (Gumbula, 2005; Christie, 2005) y más oportunidades para proteger y conservar el conocimiento indígena dentro de las comunidades (Langton \& Ma Rhea, 2005), tanto por medio de procesos de documentación como por el revitalizado interés en su utilidad futura (Anderson, 2005; Hunter, 2005), y especialmente para el fortalecimiento de las instituciones sociales, económicas y políticas de los indígenas (Ma Rhea, 2004).

También se focaliza en el compartir y transferir aspectos del conocimiento indígena entre diferentes contextos para un beneficio humano más amplio. En circunstancias de las mejores prácticas, la transferencia y/o integración del conocimiento indígena en dominios de conocimiento proporciona el debido 12 I reconocimiento y protección legal a aquellos aspectos e innovaciones del 
conocimiento que tienen un origen indígena. Por el contrario, en el caso de las peores prácticas el interés en el conocimiento indígena amenaza su integridad y lo explota a una escala aún mayor.

Dentro del medio académico, y en Australia, muchos de nosotros - seamos indígenas o no, profesores o estudiantes- desarrollamos conceptos generales y/o detallados de conocimiento, tradiciones y prácticas indígenas, a través de interpretaciones y representaciones en el idioma inglés de parte de especialistas o científicos del conocimiento occidental. Esto no quiere decir que algunos estudiantes y profesores indígenas no desarrollen conocimiento in situ en contextos indígenas. Pero sugiere que la mayor parte de la conceptualización y características que describen y sitúan el conocimiento indígena con respecto al conocimiento occidental, nos llega a través del filtro de su discurso.

Qué aspectos del conocimiento indígena son representados y la manera en que éstos son representados en este espacio reflejan un complejo conjunto de intersecciones de intereses y cuestionamientos: desde qué aspectos del conocimiento son reconocidos o valorados, qué se puede prever en términos de representación o utilidad, qué tipos de colaboración son prácticas o posibles, la capacidad de las tecnologías actuales para representar aspectos del conocimiento indígena sin destruir su integridad, hasta qué proyectos de investigación son financiados, la calidad de expertos en ambas tradiciones de conocimiento, el interés particular de científicos o sectores disciplinarios, y lo que finalmente se incluye en las bases de datos, o se publica y circula en el dominio público o el académico.

Y más importante aun, lo negativo de todos ellos: qué no es de valor o interés; qué no logra atraer financiación; qué no es investigado, documentado o publicado; qué es malinterpretado durante el proceso de abstracción del conocimiento indígena; y qué es marginalizado en la periferia a riesgo de ser excluido, no reconocido como conocimiento válido, u olvidado.

\section{La Interfaz Cultural}

En este espacio en disputa entre dos sistemas de conocimiento como es la interfaz cultural (Nakata, 1998), las cosas no son claramente blancas o negras, indígenas u occidentales.

En este espacio hay historias, políticas, economías, discursos múltiples e interconectados, prácticas sociales y tecnologías de conocimiento que 
condicionan cómo todos nosotros vemos el mundo, cómo todos llegamos a comprender nuestras cambiantes realidades cotidianas, y qué conocimiento ponemos en práctica en nuestra vida diaria. Mucho de lo que aportamos a esto es conocimiento tácito y no hablado, aquellas suposiciones a través de las cuales le damos sentido y significado a nuestro mundo cotidiano. En el terreno, en contextos prácticos donde los expertos en conocimiento indígena están en contacto directo con expertos científicos, este es el espacio de una difícil traducción entre las diferentes maneras de entender la realidad (Verran, 2005). Pero dentro del discurso intelectual, esta traducción ya ha ocurrido. El conocimiento indígena es re-presentado y re-configurado como parte del corpus "acerca" de nosotros y ya está discursivamente delimitado, ordenado y organizado por otros y su conjunto de intereses.

En la esfera académica, por tanto, llegamos a conocer "acerca" del conocimiento indígena en formas similares a la que llegamos a conocer "acerca" de las culturas y cuestiones indígenas a través de las disciplinas establecidas.

Es importante que quienes intentan introducir el conocimiento indígena a contextos de enseñanza y aprendizaje comprendan lo que sucede cuando el conocimiento indígena es conceptualizado de manera simplista y oposicionista desde el punto de vista de paradigmas científicos como todo lo que es "no-ciencia".

También es importante comprender lo que sucede cuando el conocimiento indígena es documentado de manera que lo desencarna de la gente que son sus agentes, cuando los "conocedores" de ese conocimiento se apartan de lo que se convierte en "lo conocido", en maneras que lo desarticulan de su lugar y lo separan de las instituciones sociales que sostienen y refuerzan su eficacia, y lo escinde de las prácticas que renuevan constantemente sus significados en el aquí y ahora.

Además es importante considerar qué desintegraciones y transformaciones ocurren cuando éste se redistribuye entre las categorías de clasificación occidental, cuando es manejado en bases de datos por medio de tecnologías que han sido desarrolladas en formas que se ajustan a las jerarquías, linealidad, abstracción y objetivación del conocimiento occidental, siendo todos ellos la antítesis de las tradiciones y tecnologías del conocimiento indígena.

En la carrera por documentar y registrar el conocimiento indígena en bases de datos, también es importante comprender los efectos, tanto positivos como negativos, de tal documentación. Existe literatura cada vez más abundante sobre estos temas que informan sobre cómo utilizar el conocimiento indígena 14 I con propósitos de enseñanza y aprendizaje, particularmente en la enseñanza 
universitaria de pregrado (Agrawal, 1995b; Christie, 2005; Langton \& Ma Rhea, 2005; Verran, 2005).

De tal manera, al incorporar conceptos de conocimiento indígena a áreas del currículo, al pedir a nuestros estudiantes que lean literatura al respecto o que discutan su posible aplicación a una gama de contextos profesionales, o al usarlo en formas aplicadas, debemos reconocer que estamos percibiéndolo a través de un filtro que lo posiciona para servir a nuestros objetivos educacionales, y que se basa en nuestras anteriores inversiones teóricas en el conocimiento y práctica del conocimiento.

Pero aparte de comprender estas cuestiones, existen otras consideraciones del espacio en disputa que requieren de cierta reflexión antes de analizar cualquier contenido y métodos de los estudios indígenas o la enseñanza y aprendizaje en educación superior. Éstas giran alrededor de la ubicación de los alumnos indígenas.

\section{La Ubicación del Alumno}

Independientemente de la distancia que puedan tener de lo que entendemos como "el contexto tradicional", para muchos profesores y estudiantes indígenas, la base epistemológica indígena de la construcción del conocimiento y las maneras de "hacer" conocimiento no les son completamente desconocidos.

Estas se encuentran integradas quizás no tanto en el conocimiento detallado de la tierra y del lugar de todos nosotros, ni en el conocimiento ambiental o ecológico, sino en las formas de contar historias, de hacer memoria, en la narrativa, el arte y la performance indígena; en las prácticas culturales y sociales como la manera de relacionarse con los parientes, de socializar a los niños; en las formas de pensar, de transmitir el conocimiento aun en lenguajes acriollados; y en todo lo que el popular término genérico visión del mundo abarca, etc.

Pero todos estamos también insertos en la epistemología occidental a través de la experiencia histórica, de la cristianización, el idioma inglés, de las intervenciones e interacciones con las instituciones coloniales y contemporáneas; a través de la educación formal, del uso de tecnología de la cultura popular, de la adhesión a las leyes, al mundo laboral, a los valores democráticos, etc. Esto es también familiar y reconocible y podemos aceptarlo, rechazarlo, asimilarlo, domesticarlo, usarlo, subvertirlo; pero a pesar de todo estamos constantemente relacionados con ello, mientras avanzamos en un constante 
proceso de interminables, y a menudo inconscientes, negociaciones entre estos puntos o marcos de referencia para ver, comprender y conocer al mundo.

La negociación entre estos es un proceso transformador de interminables momentos de aprender y olvidar, mezclar y separar, de tomar y descartar, de continuar y descontinuar. Participamos en estas formas de ver el mundo, de estar y actuar en él, a menudo de maneras bastante contradictorias, ambiguas o ambivalentes. Nos adherimos -con diferentes niveles de compromiso tanto en tiempo como en espacio- a varias posiciones dependiendo del momento y de las experiencias, capacidades, recursos y discursos en los que nos tenemos que basar de acuerdo a lo que está en juego para nosotros o nuestras familias o nuestra comunidad, y de acuerdo a nuestras experiencias pasadas, realidades actuales, aspiraciones y futuros imaginados. Los pueblos indígenas tienen una larga experiencia de estar ubicados en este espacio de posturas enfrentadas de la interfaz cultural.

En esta ubicación (locale), los estudiantes indígenas están discursivamente constituidos como sujetos respecto de esa "matriz de discursos extraídos que construye una conciencia de nosotros mismos, que está fuera de lo local, fuera de cómo se percibe la vida" (D. Smith, 1987, 1990, 1999). Y es a través de la comprensión de lo que constituye y es constitutivo de la experiencia indígena en esta ubicación que los catedráticos necesitan reteorizar al estudiante indígena como potencial alumno.

A fin de asegurar que quede claro: cualesquiera sean las particularidades de sus experiencias previas, los alumnos llegan a los programas universitarios, ya constituidos y posicionados discursivamente de variadas maneras para adquirir el conocimiento que ha establecido su posición.

En este contexto de aprendizaje, los discursos socio-históricos que han constituido su posición están organizados y ordenados a través de las disciplinas y el corpus, a través de un orden de las cosas occidental. Algunos de los marcos teóricos dentro de este orden han formado una posición de consenso y sentido común acerca de la comunidad indígena. El cuestionamiento del conocimiento es más fácil para los estudiantes a niveles ideológicos y de contenido dentro de esas posiciones aceptadas. El cuestionamiento también es más fácil si las áreas cuestionadas son consideradas en términos de simples intersecciones.

Sin embargo, los estudiantes indígenas a menudo sienten las contradicciones $\mathrm{y}$ tensiones cuando tienen que alinearse a una u otra de las posiciones, sobre todo cuando ven debilidades en los ejemplos y argumentos a ambos lados de la división. Es más difícil problematizar los principales conceptos 16 I teóricos y buscar aplicar un mapeo inter-subjetivo de nuestras diversas rela- 
ciones en la interfaz cultural debido a que éstas requieren una explicación de conjuntos más amplios de relaciones discursivas que van más allá de la interpretación literal del texto o los marcos teóricos dentro de un enfoque particular a un tema.

Por ejemplo, cuando utilizamos el concepto de soberanía o de autodeterminación, ¿cómo están éstos situados dentro de los conjuntos de relaciones discursivas más amplios como el discurso colonial, el discurso jurídico, el discurso de derechos, etc.? ¿Cómo ha proporcionado éste las condiciones a priori para lo que nosotros pensamos? ¿Cómo enmarca a nuestro pensamiento en una serie de áreas prácticas implicadas en ello? ¿Cómo es que nuestra adherencia a ello permite o no ciertos tipos de debate al respecto? Y, ¿̇uándo nos es posible hablar de otra cosa a fin de lograr nuestros objetivos? Por ejemplo, cuando conceptos jurídico-políticos atraviesan y constituyen complejas relaciones con el discurso antropológico y luego el de salud o educación, y luego se complican aún más con los aparatos del discurso político, gerencial y burocrático, ¿cómo se van a traer todos ellos a la superficie? ¿Cómo pueden los estudiantes suspender la manera de pensar aceptada en un área sin suspender lealtad a los intereses indígenas? ¿Pueden tomar otras posiciones sin ser etiquetados como esencialistas o asimilacionistas? Y si así lo fuera, ¿qué sería ellos?

No abrir las posiciones teóricas a discusiones más complejas ocasiona que la dinámica de la interfaz cultural esté cerrada en favor del orden de cosas occidental y a su manera de definir lo que debería ser la oposición indígena.

A menudo, los alumnos indígenas no tienen una base de experiencia completamente articulada para cuestionar el conocimiento. Con ese nivel de práctica cultural se entiende implícitamente que con frecuencia a los estudiantes indígenas les es difícil cuestionar las interpretaciones del corpus en base a lo que ellos conocen de su propia cultura. Por ejemplo, los detalles de la adopción consuetudinaria no son revelados a los jóvenes estudiantes. Puede que sepan suficiente para sentirse inquietos con la interpretación textual pero no están tan seguros de su conocimiento como para hacer algún tipo de refutación. Esta inquietud tendrá que ser suspendida para darle sentido al discurso jurídico. La elección se vuelve una entre el silencio o exponerse al reto de elementos más autorizados del corpus.

¿Cómo se puede ayudar a los alumnos indígenas a explorar su conocimiento experimental más allá del aula y a traerlo para informar cómo determinadas posiciones son cuestionadas por el compromiso con el corpus? El alumno, a fin de llegar a una posición bajo tales condiciones, debe suspender una o la otra. Ellos no pueden forjar un entendimiento sin ser llamados a 
alinearse con una posición o la otra. El alumno no tiene oportunidades de desarrollar modos de leer, formas de participar de manera crítica dentro de un discurso indígena aceptado, por lo que esto está constituido dentro de conjuntos más amplios de relaciones sociales, sin traicionar las posiciones aceptadas dentro del sistema político indígena. De este modo, es difícil manejar las tensiones inherentes al mundo cotidiano. La preparación profesional actual es inadecuada en términos de preparar a los alumnos a trabajar los dos sistemas juntos en el interés de mejores prácticas.

¿Cómo podemos, entonces, superar las complejidades de este cuestionado espacio?

\section{Una Teoría de la Posición Indígena}

Desde comienzos de los años noventa, he investigado las posibilidades de la teoría de la posición (Standpoint Theory), y en particular la posición indígena como una posición teórica que podría ser útil -algo del cotidiano y no de algún gran discurso. Sin embargo, esto no ha sido fácil. El término posición (standpoint) a menudo es substituido por perspectiva o punto de vista (viewpoint), pero estos no representan adecuadamente el uso del término en la teoría, que es bastante complejo y rebatible como enfoque teórico.

La teoría de de la posición feminista surgió en los años 70 y 80 en un intento de lidiar con el problema de articular la experiencia del mundo de las mujeres, organizada a través de sus prácticas de producción de conocimiento y que teorizaba las posturas de las mujeres como resultados lógicos y racionales, cuando en realidad eran posiciones socialmente construidas resultantes de determinadas formas de organización social, que apoyaban la posición y autoridad de los hombres sobre las mujeres (Smith, 1987).

Como método de investigación, la teoría de la posición fue utilizada por una diversidad de grupos marginalizados cuyos relatos de experiencias fueron excluidos o subyugados dentro la producción del conocimiento intelectual. Sin embargo, el análisis desde la posición de la experiencia cotidiana de la gente no es la suma de historias de experiencias vividas. No es la producción interminable de un discurso subjetivo para desorganizar relatos objetivados. Según Polhaus, esto parte de la premisa de que:

Primero, la posición social del que sabe es epistémicamente significativa; dónde está socialmente posicionado el conocedor delimitará y al mismo tiempo hará posible el conocimiento. Segundo, el conocimiento 
más objetivo no es producto de simple observación o una perspectiva desinteresada en mundo, sino que se logra intentando comprender la experiencia propia a través de una postura crítica del orden social dentro del cual el conocimiento es producido. (2002, p. 285)

Los relatos desde la posición (standpoint accounts), entonces, dependen de la reflexión y distinción entre la experiencia y la posición o standpoint (Pohlhaus, 2002). El traer al marco nuestra propia situación como "conocedores" no nos convierte en el foco del estudio pero va a "implicar investigar las relaciones sociales dentro de las que nosotros, como 'conocedores', conocemos" (p. 287). Esto también implica saber dónde mirar, y qué relaciones sociales podrían estar informando nuestro conocimiento. En gran medida, y citando nuevamente a Polhaus: "ser... [un conocedor indígena] no produce una postura crítica ya lista sobre el mundo, sino que mas bien la situación de... [conocedores indígenas] facilita preguntas de las que debemos partir a fin de producir conocimiento más objetivo" (Pohlhaus, 2002, p. 287).

La posición o standpoint no se refiere entonces a una "determinada posición social, sino mas bien es un compromiso con el tipo de preguntas encontradas alli" (Pohlhaus, 2002, p. 287), y este compromiso nos mueve a "forjar", según Harding (citado en Polhaus, 2002), una posición indígena crítica.

Por lo tanto, una posición indígena tiene que ser producida. No es una simple reflexión de la experiencia y no preexiste en el cotidiano esperando a ser revelado. No es una especie de conocimiento oculto que los pueblos indígenas poseen. Es una forma distinta de análisis, y es a la vez una construcción discursiva y un recurso intelectual para persuadir a los demás y resaltar lo que podría no haber sido el foco de atención de otros. No es determinista de ninguna verdad pero expone una base desde la cual lanza una serie de posibles argumentos para una serie de posibles objetivos. Estos argumentos aún requieren ser racionales y razonados; necesitan responder a la lógica y a los supuestos sobre los que están construidos. Los argumentos desde esta postura no pueden hacer valer una verdad que esté exenta del escrutinio de otros sobre la base de que como miembro de la comunidad, lo que digo cuenta. Se trata más bien de que lo que se diga debe poder ser sustentado.

Esto, entonces, no es una manera indígena de hacer conocimiento. Más bien argumenta a favor de lo que Harding llama "fuerte objetividad" (citado en Pohlhaus, 2002, p. 285) al aportar relatos de relaciones a las que los "conocedores" situados en posiciones sociales más privilegiadas no prestan atención. Es una forma particular de investigación. Es la explicación 
y análisis de cómo la organización social y las prácticas del conocimiento, a través de sus diversos aparatos y tecnologías de producción textual, se organizan y expresan en ese cotidiano, tal como se ve desde dentro de esa experiencia.

La experiencia vivida por gente en la interfaz no es el caso bajo investigación sino el punto de entrada para la investigación. Se trata de buscar una forma de explorar las realidades de lo cotidiano y descubrir cómo expresarlas conceptualmente desde la experiencia, en vez de depender de o emplear conceptos y categorías predeterminadas para explicar la experiencia.

La teoría de la posición no se ha desarrollado como una teoría singular o aislada sino que cristalizó a su alrededor diferentes interpretaciones de otras teorías asociadas con enfoques marxistas, el post- estructuralismo y el postmodernismo. Ha sido objeto de muchas críticas y ha sido algo desacreditada (véase Moore \& Muller, 1999; Walby, 2000). Las críticas se han dirigido a sus debilidades: el derrotismo de lo que algunos llaman a la tendencia al "relativismo epistémico", la interminable fragmentación de las categorías de diferencia, un desafortunado énfasis en "quién puede conocer" en vez de "qué puede conocerse", la preocupación con la política de la identidad y del lugar que reifica los límites entre grupos que tienen también intereses comunes, y el confinamiento de la política y la acción al reconocimiento y lugar en vez de a la redistribución y transformación. Estas debilidades necesitan ser abordadas para que se produzca información que articule modos de agencia creados en sitios locales por medio de la organización social del conocimiento y su tecnología, que den contenido sobre cómo la gente se involucra y participa en y a través de ellos.

La teoría de de la posición es en mi opinión un método de investigación, un proceso para hacer más inteligible "el corpus de conocimiento objetivado sobre nosotros" tal como se va generando y organiza nuestras realidades vividas. Veo esto como teorizar el conocimiento desde una posición particular e interesada, pero no para producir la "verdad" de la posición indígena o la terrible "verdad" de los grupos coloniales "dominantes", sino para descubrir mejor el funcionamiento del conocimiento y cómo la comprensión de los pueblos está recogida e implicada en este trabajo.

Esto es para mí un punto de partida útil para un primer principio de una teoría de la posición indígena: que los indígenas están envueltos en un espacio de conocimiento muy disputado de la interfaz cultural. Comenzaría de la premisa de que mi posición social está discursivamente constituida dentro de y es constitutiva de un complejo conjunto de relaciones sociales que se manifiestan 
a través de la organización social de mi cotidiano. Como un "conocedor" interesado, estoy buscando entender cómo es que llego a comprender -a saber, dentro de las complejidades de la interfaz, que es donde nuestra experiencia se constituye y es constitutiva del corpus.

Un segundo principio útil para la teoría de la posición indígena reconocería la agencia indígena como enmarcada dentro de los límites y las posibilidades de lo que puedo conocer desde esta posición constituida - reconocer que en la interfaz constantemente se nos pide ser al mismo tiempo continuos con una posición como discontinuos con otra (Foucault, 1972). Esto es experimentado como un tira y afloja entre las posiciones indígena y no indígena. Es decir, la común confusión al pedírsenos constantemente estar al mismo tiempo de acuerdo y desacuerdo con cualquier proposición sobre la base de una elección limitada entre las perspectivas del whitefella (hombre blanco) y blackfella (hombre negro o aborígen). En mi opinión, esto me ofrece los medios de ver mi posición en una particular relación con otros, para mantenerme en conocimiento de cómo estoy siendo posicionado y para defender una posición si fuera necesario.

Un tercer principio relacionado que podría ser útil incorporar es la idea de que las constantes "tensiones" que este tira y afloja crean son físicamente reales, y ambos informan, así como limitan, lo que se puede decir y lo que se deja sin decir en la vida diaria. El tomar en cuenta esta tensión nos ofrece opciones además de las nociones de poder estructuralista y los resultantes análisis causales. Esto, por ejemplo, nos permitirá una visión más sofisticada de las tensiones creadas entre las dualidades indígenas y no indígenas, no como la traducción literal de lo dicho o escrito en proposiciones, sino la experiencia física y memoria de tales encuentros en la vida diaria, y su inclusión como parte de la constelación de elementos a priori que informan y limitan no solo el rango sino la diversidad de nuestras respuestas.

Estos tres principios me permiten que, aún teniendo conocimiento de mi experiencia en la interfaz y pudiendo forjar una posición crítica, no esté ahí para derrocar la llamada posición dominante con argumentos simplistas de omisión, exclusión o tergiversación, sino que esté ahí para formular mejores argumentos en relación a mi posición dentro del conocimiento, y en relación a otras comunidades de "conocedores".

Todo el tiempo vemos y actuamos sobre las cosas de estas maneras. $\mathrm{Si}$ se piensa en algo como el humor indígena, este surge de esa ubicación donde formamos una comunidad alrededor de una comprensión intersubjetiva de nuestra experiencia, donde podemos entender los chistes. Observemos a los 
pobres invitados del programa cómico de Mary G. (programa australiano de radio y televisión), son los outsiders (forasteros) en este mundo de la experiencia y ellos deben entender nuestras reseñas sobre el programa y sentir lo que es no ser un "conocedor" de este mundo.

El humor y la sátira son formas particulares de análisis y comentario social. Los comediantes como Mary G. están justo ahí "haciendo" análisis social que ilumina nuestra manera de observar nuestra experiencia y que arrastra al análisis, nuestra experiencia de relacionarnos a través de un espacio donde se han constituido nuestras subjetividades compartidas. El humor indígena es una manera de ser entendido desde dentro de esta experiencia. Reconoce las tensiones y complejidades de la vida diaria y las refleja hacia este espacio y el hecho de que todos entendamos el chiste provee evidencia de nuestro conocimiento de cómo las complejidades en este espacio emergen en nuestra experiencia cotidiana. El chiste no resuelve nada pero sí articula algo sabido pero no dicho. Nos reímos porque expresa algo que reconocemos, algo que ya sabemos.

Al satirizarnos, el humor revela nuestra incompleta comprensión de cómo funciona el mundo más allá de nosotros y el misterio de sus formas. Pero en eso, también captura una dimensión importante de nuestra experiencia en esta ubicación. El humor indígena también revela la ignorancia de los outsiders sobre cómo funcionamos en y entendemos nuestro mundo, y en ese sentido todos hemos tenido una risita alegre a costa de los whitefellas. En el humor existe un escrutinio de nosotros mismos como actores en nuestro mundo y reconocimiento de ese mundo más allá de nosotros que está omnipresente y a menudo no de manera coherente y lógica desde nuestro punto de vista.

Es por esto que necesitamos una teoría de la posición que pueda generar relatos de comunidades de indígenas en espacios de conocimiento disputados, como el primer principio; que provea capacidad de agencia a la gente, como en el segundo principio; y que reconozca las tensiones cotidianas como las propias condiciones, a lo que es posible entre las posiciones indígenas y no indígenas, como el tercer principio. De esta manera podemos desplegar una posición indígena para ayudar a desentrañar y desenredarnos de las condiciones que delimitan quién, qué o cómo podemos o no podemos ser, a ayudar a vernos con cierta carga de lo cotidiano, y para ayudar a entender nuestras variadas respuestas al mundo colonial.

Entonces, ¿qué significará esto para los estudios indígenas australianos? 


\section{Estudios Indígenas Australianos}

Primero, en cuanto al sector de educación superior, debemos mantener un enfoque flexible en pos de obtener el óptimo encaje entre alumnos, aprendizaje, enseñanza y futuras profesiones, y darnos la libertad para usar todo lo que esté a nuestro alcance para lograr los mejores resultados para nuestros alumnos. Sería radicalmente tonto no usar o descartar lo que sabemos que funciona pero que no se usa sólo porque viene de tradiciones de hombres blancos.

Segundo, necesitamos reconocer que nuestros estudiantes viven en un espacio complejo y difícil y al mismo tiempo asegurarnos de no colegir nuestra comprensión de ello aquí y ahora con un pasado lejano imaginado que podría ser traído a escena para reconfigurar un futuro tradicional más simple y alejado del futuro global.

Tercero, necesitamos seguir centrados en que los futuros profesionales graduados deberán ser capaces de trabajar en terrenos complejos y cambiantes. Y comencemos con el hecho de que los alumnos indígenas están ya familiarizados con las complejidades de la interfaz cultural.

Cuarto, necesitamos diseños de currículo que aprovechen estas capacidades y que creen oportunidades para que los alumnos logren un balance de conocimientos, habilidades y procesos para explorar las fronteras interdisciplinarias. No debemos engañarnos con que un contenido correcto, por sí solo, producirá mejores resultados.

Y quinto, y esto es muy importante, los propios educadores necesitan desarrollar su erudición sobre espacios de conocimiento en disputa en la interfaz cultural y lograr para sí mismos alguna facilidad con la cual involucrar y hacer avanzar a los estudiantes en el proceso de aprendizaje.

Si mientras avanzamos con nuestro trabajo, nos aferramos a algunos de estos puntos básicos y nos reunimos anualmente para debatir qué funciona y qué no, estaríamos dando los primeros pasos para establecer los Estudios Indígenas Australianos como disciplina, con sus propias prácticas para comprometerse con y verificar el conocimiento. 


\section{Referencias}

Agrawal, A. (1995a). Dismantling the divide between Indigenous and Western knowledge. Development and Change, 26(3), 413-439.

Agrawal, A. (1995b). Indigenous and scientific knowledge: Some critical comments. Indigenous Knowledge and Development Monitor, 3, 33-41. Recuperado de http://www.nuffic.nl/ciran/ikdm/4-2/articles/agrawal.html

Agrawal, A. (1996). A sequel to the debate (2). Indigenous Knowledge and Development Monitor, 4(2), 3-4. Recuperado de http://www.uffic.nl/ciran/ ikdm/4-2/articles/agrawal.html

Anderson, J. (2005). Indigenous knowledge, intellectual property, libraries and archives: Crises of access, control and future utility. En M. Nakata \& M. Langton (Eds.), Australian Indigenous Knowledge and Libraries (pp. 85-98). Kingston, ACT: Australian Academic \& Research Libraries, Australian Library e Information Association.

Battiste, M. \& Youngblood Henderson, J. (2000). Protecting Indigenous knowledge and heritage: A global challenge. Saskatoon, SK: Purich.

Christie, M. (2005). Aboriginal knowledge traditions in digital environments. The Australian Journal of Indigenous Education, 34, 61-66.

Ellen, R. \& Harris, H. (1996). Concepts of Indigenous environmental knowledge in scientific and development studies literature: A critical assessment. Ponencia presentada en el East-West Environmental Linkages Network Workshop 3, Canterbury, United Kingdom. Recuperado de http://www. ukc.ac.uk/rainforest/SML_files/Occpap/indigknow.occpap_TOC.html

Foucault, M. (1972). The archaeology of knowledge. London: Routledge.

Gegeo, D. \& Watson-Gegeo, K. (2001). "How we know": Kwara'ae rural villagers doing Indigenous epistemology. The Contemporary Pacific, 13(1), 55-88.

Gumbula, J. Neparrnu. (2005). Exploring the Gupapuyna legacy: Strategies for developing the Galiwin'ku Indigenous Knowledge Centre. En M. Nakata \& M. Langton (Eds.), Australian Indigenous Knowledge and Libraries (pp. 25-28). Kingston, ACT: Australian Academic \& Research Libraries, Australian Library e Information Association.

Hoppers, C. (2000). The centre-periphery in knowledge production in the $21^{\text {st }}$

Century. Compare, 30(3), 283-291. 
Hunter, J. (2005). The role of information technologies in Indigenous knowledge management. En M. Nakata \& M. Langton (Eds.), Australian Indigenous Knowledge and Libraries (pp. 113-128). Kingston, ACT: Australian Academic $\&$ Research Libraries, Australian Library e Information Association.

Langton, M. \& Ma Rhea, Z. (2005).Traditional Indigenous Biodiversity-related knowledge. En M. Nakata \& M. Langton (Eds.), Australian Indigenous Knowledge and Libraries (pp. 47-72). Kingston, ACT: Australian Academic \& Research Libraries, Australian Library e Information Association.

Ma Rhea, Z. (2004). The preservation and maintenance of the knowledge of Indigenous peoples and local communities: The role of education. Ponencia presentada en la Conferencia de la Australian Association of Researchers in Education (AARE), Melbourne, Victoria. Recuperado de http://www. aare.edu.au/04pap/mar04956.pdf

Moore, R. \& Muller, J. (1999). The discourse of 'voice' and the problem of knowledge and identity in the sociology of education. British Journal of Sociology of Education, 20(2), 189-206.

Nakata, M. (1998). The cultural interface: An exploration of the intersection of Western knowledge systems and Torres Strait Islander positions and experiences. (Tesis doctoral no publicada). James Cook University, Townsville.

Nakata, M. (2002). Indigenous knowledge and the cultural interface: Underlying issues at the intersection of knowledge and information systems. IFLA Journal, 28(5/6), 281-291.

Pohlhaus, G. (2002). Knowing communities: An investigation of Harding's standpoint epistemology. Social Epistemology, 16(3), 283-293.

Russell, L. (2005). Indigenous knowledge and the archives: Accessing hidden history and understandings. En M. Nakata \& M. Langton (Eds.), Australian Indigenous Knowledge and Libraries (pp. 169-180). Kingston, ACT: Australian Academic \& Research Libraries, Australian Library e Information Association.

Smith, D. (1987). The everyday world as problematic: A feminist sociology. Boston, MA: Northeastern University Press.

Smith, D. (1990). The conceptual practices of power: A feminist sociology of knowledge. Boston, MA: Northeastern University Press.

Smith, D. (1999). Writing the social: Critique, theory and investigations. Toronto, ON: University of Toronto Press. 
Smith, L. T. (1999). Decolonizing methodologies: Research and Indigenous peoples. New York: Zed Press.

Verran, H. (2005). Knowledge traditions of Aboriginal Australians: Questions and answers arising in a databasing project. Recuperado de http://www. cdu.ed.au/centres/ik/pdf/knowledgeanddatabasing.pdf

Walby, S. (2000). Beyond the politics of location: The power of argument in a global era. Feminist Theory, 1(2), 189-206. 